\title{
On the Integration of Structure Indexes and Inverted Lists Paper Id: 616
}

\author{
Raghav Kaushik Rajasekar Krishnamurthy Jeffrey F Naughton Raghu Ramakrishnan \\ University of Wisconsin, Madison \\ \{raghav,sekar,naughton,raghu\}@cs.wisc.edu
}

\begin{abstract}
We consider the problem of how to combine structure indexes and inverted lists to answer queries over a native XML DBMS, where the queries specify both path and keyword constraints. We augment the inverted list entries to integrate them with a given structure index, and present novel algorithms for evaluating branching path expressions. Our experiments show the benefit of integrating the two forms of indexes.

We also consider the problem of evaluating path expression queries with (several alternative) extensions that incorporate relevance ranking. By integrating the above techniques with the Threshold Algorithm proposed by Fagin et al., we obtain instance optimal algorithms to push down top k computation.
\end{abstract}

\section{Introduction}

Recently, there has been a great deal of interest in the development of techniques to evaluate path expressions over collections of XML documents. In general, these path expressions contain both structural and keyword components. For example, consider the query //section/figure/title/“Graph”. This query looks for the keyword "Graph" (its keyword component) appearing at the end of a the sequence of structural containments //section/figure/title (its structural component). Several methods have been proposed for processing path expressions over graph/tree-structured XML data. These methods can be classified into two broad classes. The first involves graph traversal where the data graph is traversed using the input query $[16,24]$. The other involves information-retrieval style processing using inverted lists [7, 9, 20, 22, 30, 32, 35]. Methods have been proposed to optimize queries in the presence of both these alternatives [16, 19, 24]. In this framework, structure indexes $[14,21,25]$ have been proposed to be used as a substitute for graph traversal [24]. These structure indexes typically address only the structural component of a path expression query. To the best of our knowledge, no published work has addressed how to integrate structure indexes with information retrieval style inverted list processing for queries with both structure and keyword components. This paper attempts to fill this gap by proposing and evaluating an approach that merges structural summaries and inverted lists. Our contributions in this regard are:

- Evaluating path expressions using structure indexes and inverted lists (Section 3) We show how we can use structure indexes in conjunction with inverted lists to efficiently answer queries with both structure and value components. Our approach is to augment the inverted list entries with information derived from a structure index. Our query evaluation algorithm uses these modified entries to eliminate most inverted list joins.

- Evaluation of these techniques (Section 7) We have implemented our approach in the Niagara XML data management system [27]. Our experiments using Niagara demonstrate that we can derive substantial benefits by integrating the two forms of indexes.

While finding all documents or elements that satisfy a given path expression is a common use of path expression querying, users who specify keyword-based IR queries typically want just the $k$ most relevant answers. Several proposals have been made to incorporate the IR notion of relevance to XML queries [2, 12, 15, 28]. In this paper, we also study how integrating structure indexes with inverted lists can impact the evaluation of path expression queries, where we rank all documents that match the query and return the top $k$ documents in order of relevance, along with the specific elements that matched the query in each of these documents. We allow a broad class of relevance functions (Section 4) that covers the standard tf-idf notion of ranking. The algorithms we propose are based on Fagin et al.'s Threshold Algorithm (TA) [11]. Our setting poses novel challenges (Section 4.2), since the ranking function we allow is not necessarily monotonic [11]. Also, unlike TA which is a middleware algorithm, our focus is on the database server where additional access paths are available. This violates certain assumptions under which TA is shown to be instance optimal [11]. Our contributions here are:

- Algorithm to Merge Ranked Inverted Lists (Section 5): We adapt Fagin et al.'s Threshold Algo- 
rithm [11] to join ranked inverted lists to evaluate a single path expression. The technical challenge is due to the fact that the ranking function we use is not monotonic, as required by the algorithms in [11].

- Using Structure Indexes for top- $k$ Computation (Section 6): The above algorithm is "instance optimal" across a broad class of algorithms. However, in our domain, the presence of additional access paths leads to new algorithms that are better on some instances. The above algorithm thus fails to be instance optimal in the presence of these new access paths. However, we show that a structure index can be used in conjunction with the ranked inverted lists to design a new algorithm that is instance optimal even in the presence of these access paths. We extend this algorithm to the case when the query is a bag of simple path expressions ( $\mathrm{Sec}-$ tion 6.1), in which case, it is instance optimal for a broad subclass of ranking functions allowed. We present the results of experiments over the Niagara system [27] in Section 7.

\section{Background}

\subsection{Data Model}

Each XML document is a tree. An XML tree is a directed graph $G=\left(V_{G}, V_{T}, E_{G}\right.$, root, $\Sigma_{G}$, oid, label, ord). $V_{G}$ is the set of element nodes while $V_{T}$ is the set of text nodes, one per keyword in the XML document. $E_{T}$ is the set of edges which are constrained to induce a spanning tree over $V_{G} \cup V_{T}$. Each edge in $E_{T}$ is a parent-child edge. There is a distinguished node in $V_{G}$ called the root with no incoming edges. Nodes in $V_{T}$ have no outgoing edges, that is, they occur at the leaves of the tree. Nodes in $V_{G} \cup V_{T}$ are labeled through the label function. We assume that the labels of nodes in $V_{T}$ are the respective keywords they represent and that they are distinct from those of nodes in $V_{G}$. The labels of nodes in $V_{T}$ are placed in quotation marks to distinguish them. All nodes in $V_{G} \cup V_{T}$ are assigned unique ids through the oid function. Each node is assigned a unique ordinal number, through the ord function, which corresponds to its sibling position. We can define a total ordering on all nodes in $V_{G} \cup V_{T}$ by ordering parents before children and using the ordinal number between siblings. We refer to this as the document order. The document order corresponds to the order in which the data appears in the XML document.

Figure 1 is an example XML tree. This data represents one of the XQuery use cases available at [8]. The data represents an XML document that stores the contents of a book, in this case "Data on the Web". The book has a root book element along with tags for sections, figures, titles and paragraphs (p). These tags induce a tree structure on the document. The actual contents of the book appear at the leaf level of this tree. Some of these contents are omitted for clarity.

An XML database is a collection of XML trees/documents. The oids are constrained to be unique across the whole database. The id of the root node of a document is the document id. The whole database consists of an artificial root node with the special label ROOT that has as its children the roots of each individual document. An example would be a database of books where each book is an XML document, like the one in Figure 1.

\subsection{Path Expression Queries}

A simple path expression has the form " $s_{1} l_{1} s_{2} l_{2} \ldots$ $s_{k} l_{k}$ " where each $l_{i}$ except $l_{k}$ is a tag name, $l_{k}$ is a tag name or keyword, and each $s_{i}$ is either / or $/ /$ denoting respectively parent-child and ancestor-descendant traversal. If $l_{k}$ is a keyword, the simple path expression is called a simple keyword path expression.

A branching path expression has the form "s $s_{1} l_{1}\left[\right.$ Pred $\left._{1}\right] \quad s_{2} l_{2}\left[\right.$ Pred $\left._{2}\right] \ldots s_{k} l_{k}\left[\right.$ Pred $\left._{k}\right]$ " where each Pred $_{i}$ is an optional predicate, each $l_{i}$ except $l_{k}$ is a tag name, $l_{k}$ is a tag name or keyword, and each $s_{i}$ is either / or // denoting respectively parent-child and ancestor-descendant traversal. If $l_{k}$ is a keyword, then Pred $_{k}$ must be absent. A predicate is a simple path expression.

The result is the set of all nodes that match the path expression query. This is standard notation for path expressions, with the exception that we allow the trailing label to be a keyword.

Some example queries on the data in Figure 1 are:

1. //section//title/"web"

2. //section[/title]//figure

3. //section[/title/"web"]//figure[//“"graph"]

If a branching path expression has at least one keyword, we call it a text query. Otherwise, we call it a structure query. Queries 1 and 3 are instances of text queries while Query 2 is an instance of a structure query. The structure component of a text query $T Q$ is the structure query $S Q(T Q)$ obtained by dropping all keywords from $T Q$. For instance, the structure component of Query 3 above is Query 2.

\subsection{Structure Indexes}

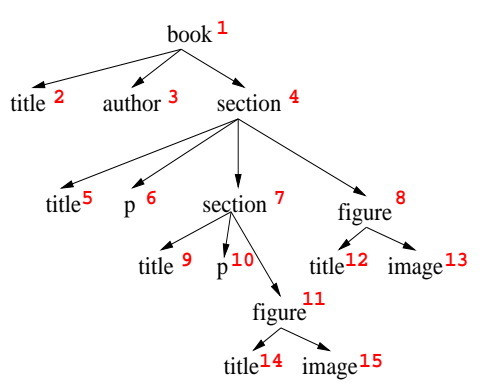

Figure 2. Example structure index 


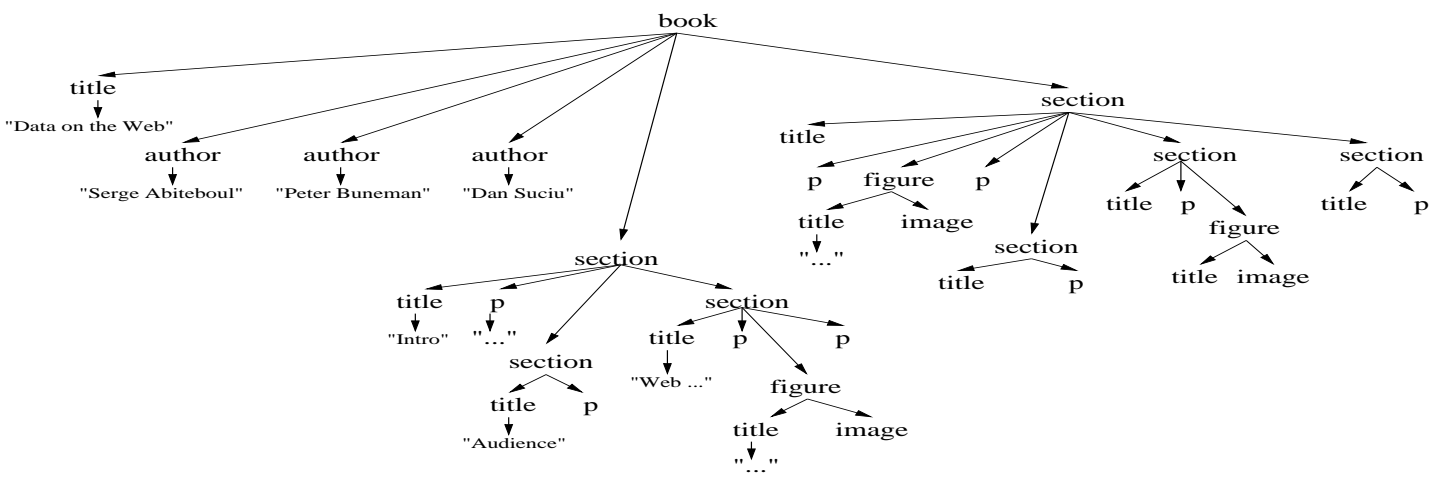

Figure 1. Sample data

A structure index $I(G)$ for the data graph $G$ corresponding to an XML database is another labeled, directed graph. The idea is to preserve all the paths in the data graph in the summary graph, while having far fewer nodes and edges. A structure index is used for query answering by associating an extent with each node in the index. In general, any partition of the element nodes defines a structure index where we (1) associate an index node with every equivalence class, (2) define the extent of each index node $n, \operatorname{ext}(n)$, to be the equivalence class that formed it and (3) add an edge from index node $A$ to index node $B$ if there is an edge from some data node in $\operatorname{ext}(A)$ to some data node in $\operatorname{ext}(B)$. Henceforth, whenever we refer to a structure index, we mean an index obtained from a partition of the data nodes through the above construction. Thus, even a simple grouping of the data nodes by label defines a structure index. Each node $A$ in the index has a unique identifier $i d(A)$. Notice that a structure index indexes only the structural part of the XML database - it ignores the text nodes.

Figure 2 shows an example structure index. The numbers shown beside each node indicate the id of that node in the index. Each element node is associated with exactly one index node inducing a partition on the data nodes.

The index result of executing a path expression $R$ on $I(G)$ is the union of the extents of the index nodes that match $R$. The extent mapping has the property that the result of any path expression $R$ on $G$ is contained in the result of $R$ on $I(G)$. For a particular path expression query $Q$, if the index result is equal to the result of $Q$ on the data graph, then $I(G)$ is said to cover $Q$.

\subsection{Inverted Lists}

Several native XML database systems [19, 27] create inverted lists on tag names and keywords. Algorithms to effectively process queries using these lists have been proposed $[30,35]$. We assume the following representation for inverted lists.

- For each element node $n$ with tag $t$, there is an entry in the corresponding inverted list of the form $<$ docid, start, end, level, indexid $>$. We denote start as n.start and likewise for the other fields.
- For each text node with label $K$, there is an entry in the corresponding inverted list of the form $<$ docid, start, level, indexid $>$.

Here, docid refers to a unique document identifier and level is the depth of the node in the tree. The start and end numbers need to satisfy the following properties:

1. For each element node $n, n . s t a r t<n$.end.

2. If (element) node $n_{1}$ is an ancestor of element node $n_{2}$, then $n_{1}$.start $<n_{2}$.start $<n_{2}$.end $<$ $n_{1}$.end.

3. If (element) node $n_{1}$ is an ancestor of text node $n_{2}$, then $n_{1}$.start $<n_{2}$.start $<n_{1}$.end.

4. If element nodes $n_{1}$ and $n_{2}$ are siblings and $\operatorname{ord}\left(n_{1}\right)<\operatorname{ord}\left(n_{2}\right)$, then $n_{1}$.end $<n_{2}$.start. A similar property holds when one or both of $n_{1}$ and $n_{2}$ are text nodes.

Path expressions can be evaluated by joining inverted lists [30,35]. In order to make these joins more efficient, auxiliary indexes have been proposed [7, 9, 16]. For example, in Niagara [16], B-Trees are used to skip parts of the inverted lists during query processing. We denote the algorithm that joins inverted lists to evaluate a path expression $p$ as $I V L(p)$. We use $I V L$ as a subroutine in our algorithm. Any of the published techniques to join inverted lists [7, 9, 20, 22, 30, 35] can be used for this procedure.

\subsection{Integrating Structure Indexes with In- verted Lists}

In order to integrate structure indexes with inverted lists, we add a new indexid field to the list entries. For a specific structure index $I$, the indexid field is set as follows.

- For an element node $n$, let the unique index node in whose extent $n$ appears be $N$. Then, n.indexid $=$ $i d(N)$.

- For a text node $n$, let the unique index node in whose extent the parent of $n$ appears be $N$. Then, $n$.indexid $=i d(N)$.

For example, for the data shown in Figure 1, with first level section elements (that is, children of the root), we store an index id of 4 . For the keyword "web" occurring under book/title, we store an index id of 2 corresponding to book/title in the index. 


\section{Evaluating Path Expression Queries}

We first present a simple scenario to illustrate how we can integrate structure indexes and inverted lists. We then present the details of how a structure index can be used to convert a simple path expression query into an inverted list scan. For branching path expressions, it turns out that the number of joins to be performed can be reduced using a structure index. Finally, we show that even for simple path expressions, performing multiple joins can out-perform a scan. We introduce the notion of extent chaining to address this issue.

\subsection{A Simple Example}

Consider the following query over the data shown in Figure 1: //section[//figure/title/"graph"] that asks for all sections that have a figure whose title contains the keyword "graph". Here, // refers to the ancestordescendant separator while / refers to the parent-child separator in the XML tree.

Evaluating the above query over a native XML database system like Niagara [27] or Timber [19] would involve joining the inverted lists corresponding to the tag names section, figure and title, and the key-word "graph". Now suppose that we have a structure index on this data, for instance the 1-Index [25], which is shown in Figure 2.

Now consider the following evaluation strategy.

1. Execute the structure component //section[//figure/title] on the structure index to obtain a set of pairs of index ids corresponding to matching $<$ section, title $>$ pairs. In this case, this step would return $S=\{<4,12\rangle,<4,14\rangle,<7,14\rangle\}$.

2. Evaluate the join section[//"graph"] using the respective inverted lists, with the additional condition that a joining < section,"graph" $>$ pair satisfies: the corresponding index id pair must be in $S$.

This strategy is correct since for any joining node pair $\left.<n_{s}, n_{w}\right\rangle$ (here, $n_{s}$ is an element node with label section and $n_{w}$ is a text node with label "graph"):

1. The fact that the parent of $n_{w}$ has index id 12 or 14 means that $n_{w}$ is under the path figure/title.

2. Since $n_{s}$ has some path to $n_{w}$ and since $n_{w}$ is under figure/title, $n_{s}$ satisfies the query.

Notice that we replace three joins with one, in the process incurring an index evaluation cost. The structure index is typically much smaller than the data. Hence, the evaluation using the structure index is likely to do well.

\subsection{Simple Path Expressions}

The algorithm for evaluating a simple path expression $q$ using a structure index $I$ is given in Figure 3. Steps 2-4 extract the structure component $q^{\prime}$ of $q$ and check whether $I$ covers $q^{\prime}$. We assume that $I$ comes with an interface to check this property. The algorithm uses $I$ only if it covers $q^{\prime}$. In this case, it evaluates $q^{\prime}$ on $I$ to obtain a set $S$ of index ids. If $t$ is a tag name, then procedure evaluateSPEWithIndex $(q, I)$

begin

I* evaluate simple path expression $q$ using index $I * /$

1. Let $q=p \operatorname{sep} t$

2. if ( $t$ is a keyword) then $q^{\prime}=p$

3. else $q^{\prime}=q$

4. if ( $I$ does not cover $\left.q^{\prime}\right)$ then

5. use $I V L(q)$ to evaluate without structure index

6. Evaluate $q^{\prime}$ on $I$

7. Let $S$ be the set of indexids returned

8. if ( $t$ is a keyword and sep is //) then

9. foreach $(i \in S)$ do

10. put all descendants of $i$ in $S$

11. Scan the inverted list for $t$ returning end only those entries $e$ where $e . i n d e x i d \in S$

\section{Figure 3. Using structure index for simple} path expression

since $I$ covers $q^{\prime}=q$, Step 11 returns exactly the entries matching $q$.

If $t$ is a keyword and sep is /, then for each entry $e$ returned in Step 11, the following holds: e.indexid $\in S$ which means that the parent of $e$ matches $q^{\prime}=p$. Hence $e$ matches $q$. The algorithm handles the case when sep is // by adding the (index) ids of descendants of all (index) nodes matching $p$ (Steps 8-10).

\subsubsection{Branching Path Expressions}

A branching path expression consists of multiple simple path expressions. We adapt the solution for simple path expressions to address each individual branch and then join appropriate lists.

We discuss the evaluation algorithm for branching path expression queries with one predicate. These ideas extend to generic branching path expressions in a straightforward manner. Queries with one predicate can be represented as $p_{1}\left[p_{2} \operatorname{sep} t\right] p_{3}$ where $p_{1}, p_{2}$ and $p_{3}$ are simple structure expressions, sep is / or // and $t$ is a keyword. Examples of queries of this kind are:

Q1 //section[/section/title/"web"]/figure/title

Q2 //section[/section//title/“web"]/figure/title

Q3 //section[/section/title/"web"]//figure/title

Q4 //section[/section/title//“web"]/figure/title

We assume that the structure index covers $p_{1}, / / p_{2}$ and $/ / p_{3}$. Depending on the presence of $/ /$ in $p_{2}, p_{3}$ or $s e p$, we get the following cases.

Case 1: None of $p_{2}, p_{3}$ and sep contains //, as in Q1.

Case 2: $p_{2}$ contains //, as in Q2.

Case 3: $p_{3}$ contains //, as in Q3.

Case 4: sep is //, as in Q4.

Cases 2,3 and 4 are not disjoint.

In addition to the usual parent-child and ancestordescendant join, we make use of the level numbers in the inverted list entries to perform level joins. For instance, section $/{ }^{2}$ title returns all title elements that are grand-children of a section element. In general, we use the notation $e_{1} /{ }^{d} e_{2}$ to denote a binary level join. This 
can be trivially implemented by comparing level numbers during an ancestor-descendant check.

In Section 3.2, we saw how we can augment the scan of an inverted list to incorporate a set of indexids. Using this idea, we were able to convert a simple path expression query into a scan of a single list. We generalize this approach to inverted list joins as follows. For a 2-way join, we use a set $S$ of indexid pairs obtained using the structure index to filter the result of the join so that only those pairs of entries whose indexids match some pair in $S$ are returned. For $n$-way joins, we use a set $S$ of $n$-tuplets of indexids. We use the special entry $T$ for an indexid to denote that any value is a match. Notice that for any inverted list join algorithm $I V L$, the modification described above is straight-forward.

We explain our algorithm by discussing how it handles Cases 1 and 2 above. Cases 3 and 4 can be similarly handled. The detailed algorithm is shown in Appendix A.

Consider Q1. Let the structure index $I$ be the one shown in Figure 1. $I$ is applicable since it covers the three expressions //section, //section/title and //figure/title. By evaluating the structure component of the query, //section[/section/title]/figure/title on $I$, we obtain a set $S$ of triplets of ids of index nodes matching section, section/title and figure/title nodes. In this case, $S=\{<4,9,12>\}$. We then evaluate the join //section[/3"web"] $/{ }^{2}$ title using $I V L$ with $S$. This strategy is correct since if $\left\langle n_{s}, n_{w}, n_{t}\right\rangle$ is a node-triplet returned finally (with corresponding labels section, "web" and title):

1. $n_{s}$ matches //section, $n_{w}$ matches //section/title/"web" and $n_{t}$ matches //figure/title.

2. $n_{s}$ is the great grand-parent of $n_{w}$ (due to a level difference of 3 ), so $\left\langle n_{s}, n_{w}\right\rangle$ matches //section[/section/title/"web"].

3. $n_{s}$ is the grand-parent of $n_{t}$ (level difference of 2), so $<n_{s}, n_{w}, n_{t}>$ matches Q1.

We now move on to Case 2. Consider Q2. The main difference from Case 1 is that there is a // as part of the predicate which means that, for Q2, the distance between a section node and a "web" node is not known in advance. Suppose evaluating the structure component of Q2 on $I$ returns a set of triplets $S$. Now, the idea is to check if we can skip the section//title join in the predicate. In order to replace $e_{1}=/ /$ section[/section//title/"web"] with $e_{2}=/ /$ section[//“web"] using $S$, we need to verify the following. If an entry $s$ (corresponding to node $n_{s}$ ) in the inverted list for section and an entry $w$ (corresponding to node $n_{w}$ ) in the inverted list for "web" satisfy $e_{2}$ and some triplet $<i_{1}, i_{2}, i_{3}>\in S$, then there must actually be a path from $n_{s}$ to $n_{w}$ matching /section//title/"web". We ensure this by checking that there is exactly one path in the structure index from $i_{1}$ to $i_{2}$. Now, we know that there is some path $p /$ "web" from $n_{s}$ to $n_{w}$ because of the containment check. By the property of structure indexes, there is a path matching $p$ from $i_{1}$ to $i_{2}$. Also, since $<i_{1}, i_{2}, i_{3}>\in S$, there is a path $p^{\prime}$ matching section//title from $i_{1}$ to $i_{2}$. But since there is exactly one path from $i_{1}$ to $i_{2}, p=p^{\prime}$. Hence, we can skip the joins. As for the /figure/title join, since there is no // separator, it can be replaced with $/{ }^{2}$ title (as in Case 1). Putting this together, we evaluate the join //section[//"web"] $/{ }^{2}$ title using $I V L$ with $S$.

\subsection{Extent Chaining}

In the above algorithm, we attempt to skip joins whenever possible using the structure index. As we will see next, it turns out that skipping joins is not always beneficial. We introduce the notion of extent chaining to address this deficiency.

Consider the query $q=/ /$ figure/title. In [9], the authors introduce algorithms to make use of B-tree indexes on the inverted lists while performing containment joins. The algorithm does not examine those parts of the inverted lists that do not participate in the join. Depending on the document structure, the join could return the figure/title nodes by examining far fewer than the total number of title entries. We perform an experiment that shows this behavior (see Section 7 for details of our experimental setup). We run the query //africa/item over the XMark [33] data where the data size is $100 \mathrm{MB}$ (see Figure 8 for the XMark schema). Performing the join using the algorithm proposed in [9] (which is implemented in our system [27]) is about 15 times faster than scanning the item inverted list. This happens since the join processes only the fraction of the item inverted list that appear under the element africa by making use of the secondary B-Tree index.

In the algorithm described in the previous section, we attempt to skip joins whenever possible. As we just saw, however, there are cases when this is not beneficial; joins could actually restrict the computation and make it more efficient. Next, we discuss how to adapt our algorithm to address this problem. The algorithm in [9] uses the fact that title is constrained to be under figure to ignore irrelevant parts of the title inverted list. Observe that we can achieve a similar effect using the set of indexids corresponding to //figure/title. This is done by chaining all title entries based on indexids. That is, each entry has a pointer to the next entry in the same document with the same indexid. We refer to this as extent chaining. Now the inverted list entry for an element and keyword has an additional next field for this pointer.

The scan of an inverted list is modified to take advantage of extent chaining as follows. The algorithm is shown in Figure 4. In step 3, we obtain the first entry in a list corresponding to a given indexid. We maintain a directory for this purpose. If the database contains only one document, for instance, then the structure index itself can store this information. For the above example over the XMark schema, using performing the scan 
procedure scanWithChaining $(L, S)$

begin

/* returns entries in list $L$ with indexid $\in S$ */

1. currEntries $=\phi$

2. foreach $(i d \in S)$ do

3. $\quad$ add fi rst entry in $L$ with indexid $i d$ to currEntries

4. while (currEntries $\neq \phi$ ) do

5. minEntry $=$ entry with minimum start number in currEntries

6. get entry $e$ in $L$ corresponding to minEntry

7. delete minEntry from currEntries

8. if (minEntry.next $\neq N U L L$ ) then

9. add minEntry.next to currEntries

10. output $e$

end

\section{Figure 4. Scan with extent chaining}

of the item inverted list using extent chaining is 1.06 times faster than performing the join //africa/item. The speedup is low in this case since the africa list contains only one entry. In Section 7, we discuss the tradeoff between performing a linear scan and using an extent chain.

Generalizing this approach to joins of inverted lists, we pass the projection of the appropriate column of $S$ (set of indexid $n$-tuples for an $n$-way join) to the corresponding scan.

\section{Ranked IR-Style Path Queries}

We now consider the role of structure indexes in supporting information retrieval style relevance-based querying over a corpus of XML documents. We first define the class of queries we consider and describe the associated relevance semantics we allow. We also discuss the challenges involved in pushing down top $k$ computation.

\subsection{Query Language and Ranking Metric}

Several proposals have been to incorporate relevancebased search into XML queries [2, 12, 15, 28]. The goal of this paper is not to define the best relevance metric over XML data, but instead it is to study the impact of merging structure indexes with inverted lists in relevance-based computation. This section is intended to define the class of queries and relevance functions we consider.

We define a relevance query to be a bag of simple keyword path expressions, analogous to the "bag of words" query model in information retrieval. Thus, we allow simple structural specification in addition to keywords.

We next examine our model for relevance computation. Let $D$ be an XML document and $p$ be a simple keyword path expression query. The relevance of $D$ to $p$ is computed using a non-negative ranking function $R(p, D)$. For a bag of simple keyword path expressions, $Q=\left\{p_{1}, \ldots, p_{l}\right\}$, we can talk about the relevance of document $D$ for each $p_{i}$. The relevance of $D$ with respect to $Q$ is computed by combining all $R\left(p_{i}, D\right)$ through a non-negative merging function $M R\left(R\left(p_{1}, D\right), \ldots, R\left(p_{l}, D\right)\right)$ (Merge-Relevance).

The ranking function $R$ must be consistent with term frequency (tf). The term frequency of $p$ in $D, t f(p, D)$, is defined to be the number of (distinct) nodes in $D$ that match $p$. As a special case, if $p$ is $/ / t$ where $t$ is a tag name or keyword, we refer to $t f(p, D)$ as the term frequency of $t . R(p, D)$ must satisfy the following property: for path expressions $p_{1}$ and $p_{2}, t f\left(p_{1}, D\right)<$ $t f\left(p_{2}, D\right) \Leftrightarrow R\left(p_{1}, D\right)<R\left(p_{2}, D\right)$. We also require that if $t f(p, D)$ is 0 , then $R(p, D)=0$. We refer to the above as the $t f$-consistency property.

The merging function $M R$ must be monotonic [11], that is, for documents $D_{1}$ and $D_{2}$, if $R\left(p_{i}, D_{1}\right) \geq R\left(p_{i}, D_{2}\right)$ for each $i$ from 1 to $l$, then $M R\left(R\left(p_{1}, D_{1}\right), \ldots, R\left(p_{l}, D_{1}\right)\right) \quad \geq$ $M R\left(R\left(p_{1}, D_{2}\right), \ldots, R\left(p_{l}, D_{2}\right)\right)$. We also require that for document $D$, if each $R\left(p_{i}, D\right)$ is 0 , then $M R\left(R\left(p_{1}, D\right), \ldots, R\left(p_{l}, D\right)\right)=0$. Any pair of ranking and merging functions that satisfy the above properties is permitted. Note that one particular example of a merge-rank function is a weighted sum of the individual ranks. Here, the weights could be inverse document frequencies (idf). Hence, the above definition of relevance permits the traditional IR notion of tf-idf based ranking.

\subsubsection{Extending Ranking to Include Proximity}

We can extend the relevance metric to account for keyword proximity when the input is a bag of simple keyword path expressions. For this purpose, we modify the relevance computation by multiplying the merge-rank function by a proximity function $\rho$. Thus, for a bag of path expressions $\left\{p_{1}, \ldots, p_{l}\right\}$, the relevance of a document $D$ is measured as $M R\left(R\left(p_{1}, D_{1}\right), \ldots, R\left(p_{l}, D_{1}\right)\right) \times \rho\left(D, p_{1}, \ldots, p_{l}\right)$. We assume that the value of the proximity function lies in the range $[0,1]$. Note that we do not assume anything about what the notion of proximity is: it could be measured by merely treating the document as a text document and using any standard IR notion of proximity, or could reflect the tree structure of the document by assigning a higher weight if there is a deeply nested element that contains all the keywords. A relevance function is said to be (1) well-behaved if $R$ is tf-consistent, $M R$ is monotonic and $\rho \in[0,1]$, (2) proximity-sensitive if it is wellbehaved and $\rho$ is not identically 1 . Note that for a single simple keyword path expression, $M R$ and $\rho$ are not relevant, instead relevance is computed using the ranking function $R$.

\subsection{Optimizing Top $k$ Computation}

The main problem in this domain is to try to find the top $k$ answers without evaluating the entire query. In order to push down the top $k$ computation, we need access paths based on relevance. We assume that for each tag name (keyword) $t$, there is an additional inverted list 


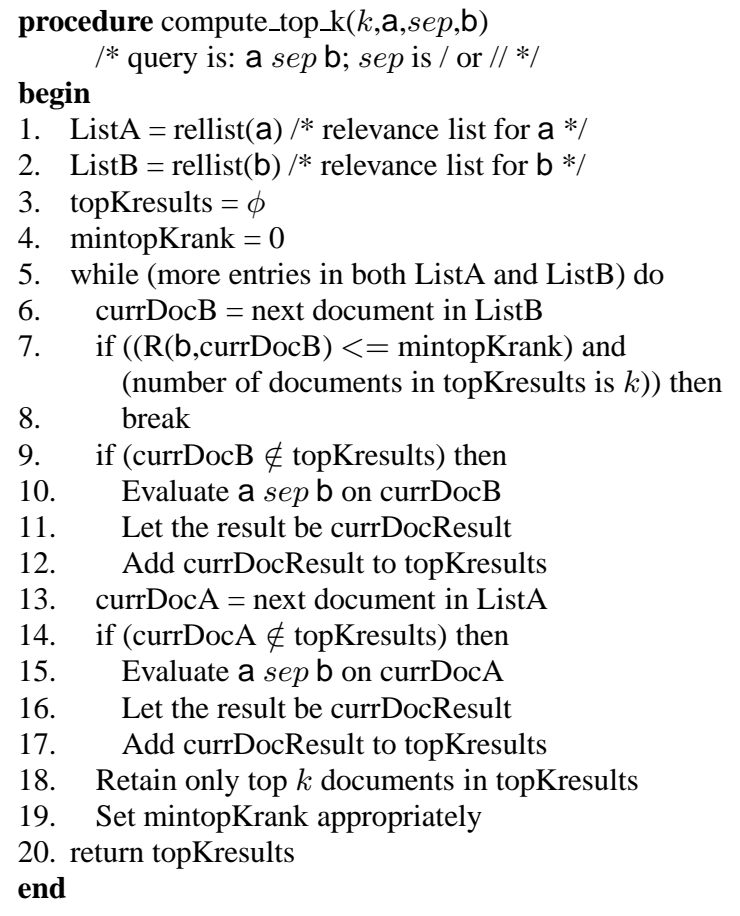

Figure 5. top $k$ algorithm for 2-way join

rellist $(t)$ where the entries within a document are in document order and the inter-document order is in descending order of relevance of $t(R(t, D))$.

Fagin et al. proposed the threshold algorithm (TA) to merge ranked lists in middleware [11]. There are two main differences in our setting.

- When we join two inverted lists, the relevance of the result is not "monotonic" in the relevance of the inputs. In other words, suppose we are evaluating $a / / b$. If we were to directly apply the threshold algorithm, then we need the following property: for documents $\mathrm{d} 1$ and $\mathrm{d} 2$, if $\mathrm{R}(\mathrm{a}, \mathrm{d} 1)>\mathrm{R}(\mathrm{a}, \mathrm{d} 2)$ and $\mathrm{R}(\mathrm{b}, \mathrm{d} 1)>\mathrm{R}(\mathrm{b}, \mathrm{d} 2)$ then $\mathrm{R}(\mathrm{a} \operatorname{sep} \mathrm{b}, \mathrm{d} 1)>\mathrm{R}(\mathrm{a} \operatorname{sep} \mathrm{b}, \mathrm{d} 2)$. This is not true in our scenario.

- TA is a middleware algorithm and is provably optimal under certain assumptions. Our focus is on the XML database server where additional access paths, like the original inverted lists, are available. These access paths violate the assumptions under which TA is proved to be optimal.

We next explore how each of these differences can be handled.

\section{Adapting TA to Inverted List Joins}

We present the details for two-way join queries. The adaptation for more joins is straight-forward. Consider the path expression a sep $\mathrm{b}$. The algorithm for this case, compute_top_k, is given in Figure 5. For steps 10 and 15, we can use any standard algorithm that merges two inverted lists [7, 9, 20, 22, 30, 35].
The procedure compute_top_k executes a sep b on a per-document basis in the process maintaining the top $k$ documents (based on relevance) among the documents processed so far in the set topKresults. When it realizes that none of the future documents can be part of the top $k$, it stops processing and returns the results. This termination condition is shown in Step 7. The maximum relevance any future document can have is the relevance of the current document in ListB. If the latter value is smaller than the relevance of the $k^{t h}$ document in topKresults, then no more documents need to be processed since the list is ordered by relevance. In addition, if we have seen all entries in either list, then the join terminates. This is so since we have executed the join for all documents containing both $\mathrm{a}$ and $\mathrm{b}$.

The main difference from the original threshold algorithm is the use of $\mathrm{R}(\mathrm{b}, \mathrm{currDoc})$ in Step 7 above. Also, unlike the original threshold algorithm, we do not assume that each document appears in every list. We handle this through the condition for the while loop in Step 5.

For a generic simple keyword path expression query $Q$, we modify compute_top_k by using the list corresponding to the result node of $Q$ to define the terminating condition like in Step 7 above, and evaluating $Q$ for each document accessed, using any standard query evaluation algorithm [7, 9, 20, 22, 30, 35]. The details are omitted for lack of space.

\subsection{Instance Optimality}

In [11], the notion of instance optimality is introduced and it is shown that the threshold algorithm is instance optimal among a certain class of algorithms. Similar results apply in our context. We use the following terminology from [11] to formalize this claim.

We consider the following modes of access to the relevance lists. For a particular list $L$, we can obtain the entries for the next document in relevance order — this corresponds to a sorted access to that document. Alternatively, we can specify a document id and ask for all entries pertaining to it. This is a random access to that document. Either access to a document returns all entries in that document. An algorithm to compute the top $k$ documents is said to make a wild guess [11] if it makes a random access on list $L$ for a document id without having seen it under sorted access under some (possibly other) list.

We now recall the notion of instance optimality [11]. Let $\mathcal{A}$ be a class of algorithms, and let $\mathcal{D}$ be a class of legal inputs to the algorithms. We assume that we are considering a particular non-negative cost measure $\operatorname{cost}(A, D)$ of running algorithm $A$ over input $D$. We say that an algorithm $B \in \mathcal{A}$ is instance optimal over $\mathcal{A}$ and $\mathcal{D}$ if for every $A \in \mathcal{A}$ and $D \in \mathcal{D}$, we have: $\operatorname{cost}(B, D)=O(\operatorname{cost}(A, D))$. In other words, there are constants $c, c^{\prime}$ such that $\operatorname{cost}(B, D) \leq c \times \operatorname{cost}(A, D)+$ $c^{\prime}$ for every choice of $A$ and $D$. We note that instance 
optimality is a stronger notion of optimality than worstcase, or even average-case optimality.

In our context, we define $\operatorname{cost}(A, D)$ of running algorithm $A$ over input $D$ to be the number of document accesses, both sorted and random, by $A$ across all lists. Computing the relevance of a document is counted as one document access. If a document is accessed on multiple lists, it is counted once per list. Similarly, if a document is accessed multiple times in the same list, it is counted once per access. We have the following theorem (the proof is omitted for lack of space).

Theorem 1: Let $q$ be a simple keyword path expression query. Let $\mathcal{D}$ be the class of all databases. Let $\mathcal{A}$ be the class of all algorithms that correctly find the top $k$ documents (and corresponding nodes) for $q$ over every database and that do not make wild guesses. Then, compute top $\_$is instance optimal over $\mathcal{A}$ and $\mathcal{D}$.

\subsection{Issues With Additional Access Paths}

Recall that we have inverted lists sorted on document id in addition to lists in relevance order. Just as in Section 3.3, where we skip parts of an inverted list within a document using secondary indexes, it is possible to skip documents during a containment join over all documents. We illustrate this next with an example. Consider the simple keyword path expression query $q=\mathrm{a} / \mathrm{b}$. Suppose the XML database has 201 documents with ids from 1 to 201. Let documents 1 to 100 have only a elements and documents 101 to 200 have only b elements. Let document 201 have an a element with child b. Consider the following algorithm for evaluating $q$.

1. Look at the first document in the two lists -1 and 101.

2. Since the document ids are different, use the larger id (in this case, 101) to seek to the first document in the list for a with document id greater than or equal to 101 .

3. The list for a is now positioned at document 201.

4. Since the document ids are still different, seek on the list for $b$ to the first document with id $\geq 201$.

5. Now both lists are positioned at 201 .

6. Perform the join over document 201.

7. Since there are no more documents on both lists, return.

This evaluation accesses only three documents. On the other hand, compute_top_k accesses all documents. The above algorithm performs efficiently on this instance due to the presence of a secondary index. Notice that in Step 3, the list for a is positioned at document 201 as a result of the random access in Step 2. But document 201 is not accessed through sorted access before this. This classifies as a wild guess and is not permitted in the class of algorithms considered in the instance optimality discussion.

We next show how we obtain an instance optimal algorithm even in the presence of these access paths. We use a structure index along with extent chaining for this purpose. procedure compute_top_k_with_sindex $(k, \mathrm{q}, \operatorname{sep}, \mathrm{b})$

/* query is: q sep b; sep is / or //, $\mathrm{q}$ is a simple path expression, $\mathrm{b}$ is a keyword */

begin

1. ListB $=$ rellist $(\mathrm{b}) / *$ relevance list for $\mathrm{b} * /$

2. if $($ sep is $/)$ then

3. indexidList $=$ list of ids of index nodes matching $\mathrm{q}$

4. else /*sep is //*/

5. indexidList $=$ list of ids of index nodes matching $\mathrm{q}$ and their descendants in the structure index

6. topKresults $=\phi$

7. mintopKrank $=0$

8. while (more entries in ListB) do

9. $\quad$ currDoc $=$ next document in ListB with at least one entry $e$ such that $e$.indexid $\in$ indexidList (use extent chaining)

10. if $((\mathrm{R}(\mathrm{b}$, currDoc $)<$ mintopKrank $)$ and (number of documents in topKresults is $k$ )) then

11. break

12. currDocResult $=\{e: e \in$ ListB corresponding to currDoc and e.indexid $\in$ indexidList $\}$ (use extent chaining)

13. Add currDocResult to topKresults

14. if (topKresults has $k+1$ documents) then

15. remove document with least relevance

16. Set mintopKrank appropriately

17. return topKresults

end

Figure 6. top $k$ algorithm using structure index

\section{Instance Optimality with a Structure In- dex}

We show how structure indexes can be used to obtain an instance optimal algorithm even in the presence of these access paths. We first consider the case when the relevance query has a single path expression. We then extend our algorithm in Section 6.1 to the case when the relevance query is a bag of path expressions.

The evaluation of a simple keyword path expression $Q=q \operatorname{sep} b$ using a structure index $I$ that covers it results in a scan on the inverted list of $b$ with a set $S$ of indexids. The algorithm for computing the top $k$ documents in this case is shown in Figure 6. We modify the idea of extent chaining introduced in Section 3.3 to chain all entries in the relevance inverted lists with the same indexid even across documents. Thus, each entry has a pointer to the next entry with the same indexid even if it is not in the same document. We observe the following about this algorithm.

- Steps 2-5 initialize the indexidList appropriately depending on whether sep is / or //.

- The terminating condition in Step 13 is similar to the one in the procedure compute_top_k.

- The evaluation of currDocResults in Step 15 (for a single document) can be performed using intradocument extent chaining described in Section 3.3.

- In Step 12, we use inter-document extent chaining to advance to the next document in ListB having at 
least one match for $\mathrm{q}$ sep $\mathrm{b}$.

\section{Implementation Note}

When performing a scan using extent chaining, to get the next entry in the list (like in Step 5 in Figure 4), we might need to compare the next pointers of more than one entry and find which of them appears first in the relevance list. The relative position of two documents in a relevance list cannot be obtained by comparing their document ids. Hence, we introduce relevance document ids (reldocids). All documents appearing in a relevance list are assigned reldocids based on their order in the list. The next pointer of an entry contains the reldocid and start number of the next entry with the same indexid. Using the reldocids, we can compare the next pointers of more than one entry. An entry in the relevance list for a tag name is of the form: <reldocid, start, end, level, indexid, docid, next_reldocid,next_start>. An entry for a keyword is the same except for the absence of end. We emphasize that the reldocid is used only for extent chaining. In particular, when we talk about document ids, we refer to the unique document id that is common to a document across all lists.

\section{Instance Optimality}

In addition to the sorted and random access modes on the relevance lists, we allow sorted and random access on the inverted lists sorted on document id. We modify the wild guess definition to obtain what we call a strict wild guess, by excluding the following: (1) random access on any list to first document with id $\geq$ a given id, and (2) random access on any list $L$ to a document with reldocid obtained from the next field of an entry (in $L$ ). Cost is measured in the same way as in Section 5.1. In particular, the index evaluation cost is not counted for the purpose of this discussion.

Theorem 2: Let $q$ be a simple keyword path expression query. Let $\mathcal{D}$ be the class of all databases such that $q$ is covered by structure index I. Let $\mathcal{A}$ be the class of all algorithms that correctly find the top $k$ documents (and corresponding nodes) for $q$ over every database and that do not make strict wild guesses. Then, compute_top_k_with_sindex is instance optimal over $\mathcal{A}$ and $\mathcal{D}$.

\subsection{Extension to Bag of Simple Keyword Path Expressions}

We now extend the above algorithm to the case when the query is a bag of simple keyword path expressions; intuitively, this corresponds to the class of IR queries with multiple keywords. Consider the evaluation of query $Q=\left\{p_{1}, p_{2}\right\}$. Using the structure index, we can convert each $p_{i}$ to a scan on the appropriate relevance list. What remains now is to merge these relevance lists and apply the relevance function for $Q$ (which merges the relevances of the $p_{i}$ and takes a product with the procedure compute_top_k_bag( $k, p_{1} \operatorname{sep}_{1}$ a, $p_{2} s e p_{2}$ b)

begin

1. List $\mathrm{A}=$ rellist $(\mathrm{a}) / *$ relevance list for $\mathrm{a} * /$

2. $\operatorname{ListB}=$ rellist $(\mathrm{b}) / *$ relevance list for $\mathrm{b} * /$

3. Obtain indexidListA and indexidListB appropriately using the structure index

//as in Steps 2-8 of compute_top_k_withsindex

4. topKresults $=\phi$

5. mintopKrank $=0$

6. while (more entries in either ListA or ListB) do

7. currDocA = next document in ListA, as per extent chaining

8. currDocB = next document in ListB, as per extent chaining

9. $R_{a}=\mathrm{R}(\mathrm{a}$, currDocA $)$

10. $R_{b}=\mathrm{R}(\mathrm{b}$, currDocB)

11. if ( $\left(\mathrm{MR}\left(R_{a}, R_{b}\right)<=\right.$ mintopKrank $)$ and (number of documents in topKresults is $k$ )) then

12. break

13. if (currDocA $\notin$ topKresults) then

14. Evaluate the two path expressions on currDocA

15. Let the result be currDocResult

16. Add currDocResult to topKresults

17. Do similarly for currDocB

18. Retain only top $k$ documents in topKresults

19. Set mintopKrank appropriately

20. return topKresults

end

\section{Figure 7. top $k$ algorithm for 2 simple key- word path expressions}

proximity function $\rho$ ). This merge is similar to the merge algorithm in Figure 5. The algorithm is shown in Figure 7. Since the merging function is monotonic and $\rho$ lies in the range $[0,1]$, this algorithm can easily be shown to be correct for all well-behaved relevance functions. This algorithm naturally extends when $Q$ has more than two simple path expressions.

We show that for the special case when the relevance function is not proximity-sensitive, this algorithm is instance optimal for an interesting class of bag queries, over the class of algorithms that do not make strict wild guesses, as defined above. A bag B of simple path expressions is defined to be disjoint if the trailing terms of no two simple path expressions in B are the same. For example, the bag \{book//“XML",author/“Abiteboul” $\}$ is disjoint while the bag $\{$ book//“XML",article//“XML” $\}$ is not.

Theorem 3: Let $Q=\left\{q_{1}, q_{2}, \ldots, q_{l}\right\}$ be a bag of simple keyword path expression queries. Let $\mathcal{D}$ be the class of all databases such that each $q_{i}$ is covered by structure index I. We have the following.

1. For any database $D \in \mathcal{D}$, compute top $\_\_b a g$ correctly returns the top $k$ documents over any given well-behaved relevance function.

2. Suppose that $Q$ is a disjoint bag and that the relevance of a document is computed using a function that is well-behaved but not proximity-sensitive. Let $\mathcal{A}$ be the class of all algorithms that correctly find the top $k$ documents (and corresponding nodes) for $Q$ over every database and that do not make strict 


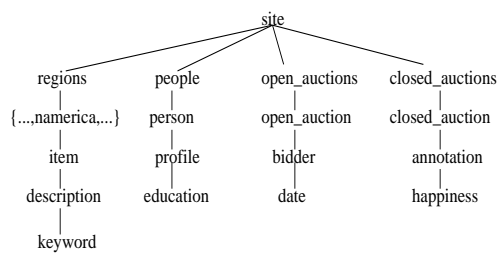

Figure 8. XMark Schema

wild guesses. Then, compute top $\_\_b a g$ is instance optimal over $\mathcal{A}$ and $\mathcal{D}$.

The optimality of the algorithm is open for proximitysensitive relevance functions.

\section{Experimental Results}

We have implemented the above algorithms as part of the Niagara XML database system [27]. We present the results of some preliminary experiments that yield a sense for the efficacy of our techniques. We first present our results for evaluating branching path expression queries using structure indexes and inverted lists. We then move on to relevance queries. Our experiments are run on a Linux Workstation with 256MB of RAM. We use a 16MB buffer pool.

\subsection{Evaluation of Branching Path Queries}

We use the XMark XML-benchmark data [33] for this set of experiments. This data models an auction site. The element relationships relevant to this paper are shown in Figure 8. The tag names are self-explanatory. The data size is $100 \mathrm{MB}$. The structure index we use is the 1Index [25]. A study of how the choice of structure index impacts performance is future work. We report the performance results for four queries involving structure and value constraints based on warm buffer pool times. We measure the speedup, defined to be the ratio of the (execution) time taken in the absence of a structure index (inverted list join) to the time taken by our algorithm. In the presence of alternative query plans, we use the execution time corresponding to the best plan. Table 1 shows the queries and the respective speedups. We discuss in Section 8 what happens when we use other inverted list join algorithms proposed in the literature.

The main observations to be made from the above numbers are:

- The benefit of using a structure index in conjunction with inverted lists is considerable with speedups of as high as about 43 times for simple path expressions and about 7 times for branching path expressions.

- The speedup obtained is dependent on the number of joins saved. At the extreme, if we remove all joins replacing them with a scan, then the speedup obtained is highest. Thus, for the first query above which is a simple path expression, the speedup obtained is highest.
Using a secondary index while performing an inverted list join adds an overhead, especially when we skip only small portions of the lists. Using an extent chain instead of a linear scan may exhibit similar behavior. In a separate set of experiments, we compare the performance of extent chaining as opposed to performing a linear scan by varying the query selectivity. Our conclusion is that if the selectivity is below a certain threshold, using extent chaining is beneficial; otherwise, a modified scan algorithm that judiciously uses the extent chain only if it finds half a page of contiguous entries that do not belong to the query result works best. The worst case cost of this algorithm in our experimental setup was $20 \%$ more than the cost of a linear scan, and the best case benefits were similar to the original extent chain based scan. Details of this study are omitted for lack of space.

\subsection{Relevance Queries}

We have implemented the compute_top_k_withsindex algorithm shown in Figure 6. Recall that this is an instance optimal algorithm for relevance queries consisting of a single (simple) path expression. We wish to study the benefit obtained through two aspects of this algorithm - the early termination condition and extent chaining. Consider a query $q=p / / t$. In the scenario where $t$ occurs in many documents but very few of these match $q$, extent chaining is likely to yield significant performance benefit. On the other hand, if $t$ occurs in many documents and most of these occurrences match $q$, the early termination condition is likely to contribute.

To study this, we use NASA's public astronomy XML archive [4]. We pick a different data set for this study since we want a data set with multiple files to make ranked queries meaningful. The data has $2443 \mathrm{XML}$ documents with a total size of about 33MB. We consider two queries - Q1 and Q2 - that search for occurrences of a particular word "photographic" under two different paths $p_{1}=$ keyword and $p_{2}=$ dataset respectively. There are very few occurrences of "photographic" under keyword, while all occurrences are under dataset.

Table 2 shows the results of our experiment. For each value of $k$, we report the speedup obtained through our algorithm, measured as the ratio of the time taken to fully execute the query on the database to the time taken by our algorithm. We also report the number of documents accessed by our algorithm.

We observe first of all that there is a significant benefit to be obtained by pushing down the top $k$ computation, instead of evaluating the query completely and then extracting the top $k$ results. For Q1, notice that the number of documents accessed by our algorithm varies very little with $k$. This indicates that the benefit is chiefly through extent chaining. On the other hand, for $\mathrm{Q} 2$, the number of documents accessed increases linearly with $k$, showing the role played by the early termination condition. 


\begin{tabular}{|l|c|c|}
\hline \multicolumn{1}{|c|}{ Query in English } & Path expression & Speedup \\
\hline Find occurrences of “attires” under item descriptions & //item/description//keyword/“attires”] & 43.3 \\
\hline Find open auctions that had a bid in 1999 & //open_auction[/bidder/date/“1999”] & 6.85 \\
\hline Find the persons who attended Graduate school & //person[/profi le/education/“Graduate’] & 5.06 \\
\hline $\begin{array}{l}\text { Find closed auctions where the happiness level } \\
\text { was 10 }\end{array}$ & //closed_auction[/annotation/happiness/“10”] & 3.12 \\
\hline
\end{tabular}

Table 1. Speedups Using Structure Index

\begin{tabular}{|l||c|c||c|c|}
\hline Value of $k$ & Speedup for Q1 & $\begin{array}{c}\text { \# Documents Accessed } \\
\text { by our algorithm }\end{array}$ & Speedup for Q2 & $\begin{array}{c}\text { \# Documents Accessed } \\
\text { by our algorithm }\end{array}$ \\
\hline 1 & 16.04 & 20 & 18.07 & 2 \\
\hline 5 & 14.92 & 25 & 10.38 & 6 \\
\hline 10 & 14.53 & 25 & 8.13 & 10 \\
\hline 50 & 12.42 & 27 & 3.67 & 51 \\
\hline 100 & 12.42 & 27 & 2.15 & 101 \\
\hline 300 & 12.42 & 27 & 1.7 & 301 \\
\hline
\end{tabular}

Table 2. Results for top $k$ queries

\section{Related Work}

Several methods have been proposed for processing queries over graph-structured XML data. These methods can be classified into two broad classes. The first involves graph traversal where the data graph is traversed using the input query [16, 24]. The other involves information-retrieval style processing using inverted lists [7, 9, 20, 22, 30, 32, 35]. Methods have been proposed to optimize queries in the presence of both these alternatives $[16,19,24]$. In this framework, structure indexes such as the ones proposed in [14, 21, 25] have primarily been used as a substitute for graph traversal [24]. However, to the best of our knowledge, no published work has addressed how to integrate structure indexes with information retrieval style inverted list processing. This is the focus of our paper. We note that our techniques apply irrespective of which specific structure index and inverted list join algorithm is used. The technique we propose is similar to the algorithm proposed in [34] using path ids in the context of evaluating branching path expression queries over XML data stored in an RDBMS. That algorithm is correct for nonrecursive data sets - it turns out that it does not give the correct result when the input XML data has an ancestor and descendant element with the same tag name. Since path ids are a special form of structure indexes [25], and their technique is based on containment joins, the solution presented in this paper can be easily applied to tbeir context.

Several techniques for inverted list joins have been proposed $[7,9,20,22,30,35]$. While our technique works when any of these algorithms used to join inverted lists (i.e., as $I V L$ ), the actual speedup obtained depends on the specific algorithm. We now discuss how the results reported in Section 7 vary with the specific join algorithm. The inverted list join algorithms can be broadly classified as follows: merge-based algorithms [22, 35], stack-based algorithms [7, 30] and extensions to these using secondary indexes [7, 9, 16, 20]. In Niagara [16], the inverted list join algorithm is a merge-based algorithm that uses auxiliary B-Tree indexes to skip parts of the inverted lists. We use this implementation to show the benefit of integrating structure indexes. Since the parts of the XMark dataset relevant to the above queries are not recursive (i.e., no two elements where one is an ancestor of the other have the same tag name), the stackbased algorithms [7, 30] do not add any benefit to mergebased inverted list processing for this dataset. Indeed, the speedups we report in Section 7 are relevant when any of the above algorithms is used, except for the XRTree [20]. We defer a study of this speedup to future work.

Several proposals have been made for ranked search over a corpus of document databases combining keyword and structure components [17, 28]. Recently, in $[2,3,12,29,31]$, query languages that integrate information retrieval related features such as ranking and relevance-oriented search into XML queries have been proposed. Techniques to evaluate these ranked queries are also proposed in $[2,3,29,31]$. A survey of commercial XML search engines is available in [23]. In [26], the problem of ranking SGML documents using term occurrences is considered. The focus of our paper is not on defining the best query model over XML documents. Instead our interest is in understanding the role of structure indexes in efficiently push down top $k$ computation. To the best of our knowledge, none of these previous techniques uses structure indexes of the kind we describe in our paper. Several previous projects have dealt with supporting ranked keyword search, like $[1,18]$ over structured databases, $[5,10,13]$ over graph-structured data, $[6,11]$ over web sources and [15] over XML data. Our query language permits a structural component in addition to keyword specification.

\section{Conclusions}

We presented methods of integrating structure indexes and inverted lists. By appropriately augmenting inverted list entries, we showed how inverted list joins could be replaced with an index navigation when evaluating branching path queries. Our experiments on the 
Niagara native XML database system showed the efficacy of this approach.

Throughout our discussion, we assumed that an XML document has two parts - one that is summarized by the structure index and one that is not. We used element nodes and text nodes to identify these parts. There can be several ways of defining these parts. For instance, the values of some text nodes can be captured in the structure index by treating them as tag names. The techniques presented in this paper are applicable irrespective of how we arrive at these two parts. However, this paper is not about how we define these parts. This is an interesting area for future work. Other such areas include looking at the tradeoffs involved in picking a structure index and integrating multiple structure indexes with inverted lists.

We also considered the evaluation of top $k$ queries over XML documents. We showed how the augmented "relevance" inverted lists combined with adaptations of the Threshold algorithm proposed by Fagin et al. yields instance optimal algorithms for pushing down top $k$ computation. In our context, the ranking function is nonmonotonic and there are additional access paths available. Using a structure index, we were able to successfully adapt the Threshold algorithm to proximitysensitive ranking functions. When the ranking function is well-behaved, our algorithm is instance-optimal. While we presented algorithms for tree structured data, they can be extended to work for graph-structured data. Several avenues remain for future work. For instance, the problem of running structured queries over hyperlinked XML documents remains open.

\section{References}

[1] S. Agrawal, S. Chaudhuri, and G. Das. DBXplorer: A system for keyword-based search over relational databases. In Proceedings of ICDE, 2002.

[2] S. Al-Khalifa, C. Yu, and H. V. Jagadish. Querying structured text in an xml database. In SIGMOD, 2003.

[3] S. Amer-Yahia, S. Cho, and D. Srivastava. Tree pattern relaxation. In $E D B T, 2002$.

[4] X. A. A. at NASA. XML astronomy archive at NASA. http://xml.gsfc.nasa.gov/archive.

[5] G. Bhalotia et al. Keyword searching and browsing in databases using BANKS. In Proceedings of ICDE, 2002.

[6] N. Bruno, L. Gravano, and A. Marian. Evaluating top-k queries over web-accessible databases. In ICDE, 2002.

[7] N. Bruno, N. Koudas, and D. Srivastava. Holistic twig joins Optimal XML pattern matching. In Proceedings of SIGMOD, 2002.

[8] D. Chamberlin, D. Florescu, J. Robie, J. Siméon, and M. Stefanescu. XQuery: A query language for XML. World Wide Web Consortium, http: / / www . w3 . org / TR/xquery, Feb 2000.

[9] S. Chien, Z. Vagena, D. Zhang, V. J. Tsotras, and C. Zaniolo. Effi cient structural joins on indexed XML documents. In Proceedings of $V L D B, 2002$.

[10] S. Dar et al. DTL's Dataspot: database exploration using plain language. In $V L D B, 1998$.

[11] R. Fagin, A. Lotem, and M. Naor. Optimal aggregation algorithms for middleware. In Proceedings of PODS, 2001.

[12] N. Fuhr and K. Grobjohann. XIRQL: A language for information retrieval in XML documents. In Proceedings of SIGIR, 2001.
[13] R. Goldman et al. Proximity search in databases. In Proceedings of $V L D B, 1998$.

[14] R. Goldman and J. Widom. Dataguides: Enabling query formulation and optimization in semistructured databases. In TwentyThird International Conference on Very Large Data Bases, pages 436-445, 1997.

[15] L. Guo, F. Shao, C. Botev, and J. Shanmugasundaram. XRANK: Ranked keyword search over XML documents. In Proceedings of SIGMOD, 2003.

[16] A. Halverson et al. Mixed mode XML query processing. In $V L D B, 2003$.

[17] M. Hearst and C. Plaunt. Subtopic structuring for full-length document access. In Proceedings of SIGIR, 1993.

[18] V. Hristidis, L. Gravano, and Y. Papakonstantinou. Effi cient irstyle keyword search over relational databases. In $V L D B, 2003$.

[19] H. Jagadish et al. TIMBER: A native XML database. VLDB Journal, 2003.

[20] H. Jiang, H. Lu, W. Wang, and B. C. Ooi. XR-Tree: Indexing XML Data for Effi cient Structural Joins. In ICDE, 2003.

[21] R. Kaushik, P. Bohannon, J. Naughton, and H. Korth. Covering indexes for branching path queries. In Proceedings of SIGMOD, 2002.

[22] Q. Li and B. Moon. Indexing and querying XML data for regular path expressions. In Proceedings of VLDB, 2001.

[23] R. Luk et al. A survery of search engines for XML documents. In SIGIR Workshop on XML and IR, 2000.

[24] J. McHugh and J. Widom. Query optimization for XML. In Proceedings of VLDB, 1999.

[25] T. Milo and D. Suciu. Index structures for path expressions. In ICDT: 7th International Conference on Database Theory, 1999.

[26] S. Myaeng et al. A fexible model for retrieval of SGML documents. In SIGIR, 1998.

[27] J. Naughton et al. The Niagara internet query system. IEEE Data Engineering Bulletin, 24(2), 2001.

[28] G. Navarro and R. Baeza-Yates. Proximal nodes: A model to query document databases by content and structure. ACM Transactions on Information Systems, 15(4), 1997.

[29] T. Schlieder and H. Meuss. Result ranking for structured queries against XML documents. In DELOS Workshop on Information Seeking, Searching and Querying in Digital Libraries, 2000.

[30] D. Srivastava, S. Al-Khalifa, H. Jagadish, N. Koudas, J. Patel, and Y. Wu. Structural joins: A primitive for effi cient XML query pattern matching. In Proceedings of ICDE, 2002.

[31] A. Theobald and G. Weikum. The index-based XXL search engine for querying XML data with relevance ranking. In $E D B T$, 2002.

[32] W. Wang, H. Jiang, H. Lu, and J. X. Yu. PBiTree Coding and Effi cient Processing of Containment Joins. In ICDE, 2003.

[33] Xmark: The xml benchmark project. http://monetdb.cwi.nl/xml/index.html.

[34] M. Yoshikawa, T. Amagasa, T. Shimura, and S. Uemura. XRel: a path-based approach to storage and retrieval of XML documents using relational databases. ACM Transactions on Internet Technology (TOIT), 1(1):110-141, 2001.

[35] C. Zhang, J. Naughton, D. DeWitt, Q. Luo, and G.Lohman. On supporting containment queries in relational database management systems. In Proceedings of SIGMOD, 2001. 


\section{A Evaluating Branching Path Queries us- ing Structure Indexes and Inverted Lists}

procedure evaluateWithIndex $(q, I)$

begin

1. Let $q=p_{1}\left[p_{2}\right.$ sep $\left.t\right] p_{3}$

2. if ( $I$ does not cover $p_{1}$ or $/ / p_{2}$ or $\left./ / p_{3}\right)$ then

3. use $\operatorname{IVL}(q)$ to evaluate without structure index

4. Let $l_{1}, l_{3}$ be the trailing tag names of $p_{1}, p_{3}$ respectively

5. Let $d 2=$ number of tag names in $p_{2}+1$

6. Let $d 3=$ number of tag names in $p_{3}$

7. Let $p_{2}^{\prime}=/{ }^{d 2} t$

8. Let $p_{3}^{\prime}=/{ }^{d 3} l_{3}$

9. Evaluate $q^{\prime}=p_{1}\left[p_{2}\right] p_{3}$ on $I$

10. Let indexTriplets $=\left\{\left\langle i_{1}, i_{2}, i_{3}\right\rangle: i_{1}, i_{2}, i_{3}\right.$ match $l_{1}, l_{2}, l_{3}$ respectively in the evaluation of $q^{\prime}$ on $\left.I\right\}$

11. if ( sep is //) then $/ *$ matches case $4 * /$

12. foreach ( $<i_{1}, i_{2}, i_{3}>\in$ indexTriplets $)$ do

13. foreach $\left(i_{2}^{\prime}\right.$ descendant of $\left.i_{2}\right)$ do

14. add $<i_{1}, i_{2}^{\prime}, i_{3}>$ to indexTriplets

15. $p_{2}^{\prime}=/ / t$

16. if ( $q$ matches case 2$)$ then

17. skipJoins $2=$ true $/ *$ verify if we can use index to skip joins in $p_{2} *$ /

18. foreach $\left(<i_{1}, i_{2}, i_{3}>\in\right.$ indexTriplets $)$ do

19. skipJoins $2=$ exactlyOnePath $\left(i_{1}, i_{2}\right)$

20. if (skipJoins2 is true) then $p_{2}=/ / t$

21. else $p_{2}=p_{2} \operatorname{sep} t$

22 . if ( $q$ matches case 3 ) then $/ *$ symmetric to case $2 * /$

23. skipJoins $3=$ true $/ *$ verify if we can use index to skip joins in $p_{3} * /$

24. foreach ( $<i_{1}, i_{2}, i_{3}>\in$ indexTriplets) do

25. skipJoins $3=$ exactlyOnePath $\left(i_{1}, i_{3}\right)$

26. if (skipJoins 3 is true) then $p_{3}^{\prime}=/ / l_{3}$

27. else $p_{3}^{\prime}=p_{3}$

28. if (skipJoins 2 is false) then

29. foreach $\left(<i_{1}, i_{2}, i_{3}>\in\right.$ indexTriplets $)$ do

30. $\quad i_{2}=\top$

31. if (skipJoins 3 is false) then

32. foreach ( $<i_{1}, i_{2}, i_{3}>\in$ indexTriplets $)$ do

33. $\quad i_{3}=\top$

34. Perform the join $l_{1}\left[p_{2}^{\prime}\right] p_{3}^{\prime}$ using $I V L$ with indexTriplets and return the results end

procedure exactlyOnePath $\left(i_{1}, i_{2}\right)$

/* $i_{1}$ and $i_{2}$ are nodes in $I * /$

$/ *$ returns true if there is exactly one path from $i_{1}$ to $i_{2} * /$ begin

1. Go backwards from $i_{2}$ to $i_{1}$ in $I$

2. Let $p$ be a path from $i_{1}$ to $i_{2}$

3 . if (any node in $p$ has $>$ one in-coming edge in $I$ ) then

4. if (sources of $>1$ are reachable from $i_{1}$ ) then

5. $\quad / *$ found $2 \mathrm{p}$

6. if ( $i_{1}$ is part of a cycle) then

7. return false

8. return true

end

Figure 9. Evaluation algorithm for branching path expressions using structure index 